\title{
Identification of floR Variants Associated with Tn4371-Like Integrative and Conjugative Elements in Human Clinical Pseudomonas Aeruginosa Isolates
}

\section{Changrui Qian}

26453-Wenzhou Medical University

Hongmao Liu

26453-Wenzhou Medical University

Wei Lu

26453-Wenzhou Medical University

Jiawei Cao

26453-Wenzhou Medical University

Junwan Lu

26453-Wenzhou Medical University

Aifang Li

117926-Lishui Central Hospital and Fifth Affiliated Hospital of Wenzhou Medical College

Xinyi Zhu

26453-Wenzhou Medical University

\section{Kai Shen}

26453-Wenzhou Medical University

Haili Xu

26452-Wenzhou Medical University Second Affiliated Hospital

Qianqian Chen

26453-Wenzhou Medical University

Wangxiao Zhou

26453-Wenzhou Medical College

Hongyun Lu

The second people hospital of Tongxiang City

Hailong Lin

26453-Wenzhou Medical University

\section{Xueyao Zhang}

26453-Wenzhou Medical University

Qiaoling Li

26453-Wenzhou Medical University 


\section{Xi Lin}

26453-Wenzhou Medical University

\section{Kewei Li}

26453-Wenzhou Medical University

\section{Hailin Zhang}

26452-Wenzhou Medical University Second Affiliated Hospital

\section{Teng Xu}

117847-Inner Mongolia Baotou City Central Hospital

Qiyu Bao ( $\sim$ baoqy@genomics.cn )

Wenzhou Medical University https://orcid.org/0000-0002-9550-8266

\section{Research}

Keywords: floR variants, integrative and conjugative elements, Pseudomonas aeruginosa, Human pathogen

Posted Date: September 4th, 2020

DOI: https://doi.org/10.21203/rs.3.rs-67840/v1

License: (c) (i) This work is licensed under a Creative Commons Attribution 4.0 International License. Read Full License 


\section{Abstract}

Background: Florfenicol is widely used to control respiratory diseases and intestinal infections in food animals. However, dramatic and serious florfenicol resistance in various clinical strains was reported. As a key resistance gene for florfenicol, floR has often been associated with mobile genetic elements. To analyze the potential transmission of floR, we investigated floR gene in Pseudomonas aeruginosa isolates from human clinical samples and characterize two floR variants, floR-T1 and floR-T2.

Methods: Pooled genomic DNA sequencing and PCR were used to analyze the floR gene in $P$. aeruginosa. The floR variants were cloned, and the minimum inhibitory concentrations (MICs) were determined. Quantitative RT-PCR was used to compare the gene expression of the two floR variants in TL1285 with or without florfenicol/chloramphenicol. Whole-genome sequencing was used to identify the genetic context of the floR variants in TL1285.

Results: Three types of $f l o R$ variants (designated $f l o R$, floR-T1 and $f l o R-T 2$ ) were identified in the clinical $P$. aeruginosa isolates, and floR-T1 was the most prevalent variant. The positive rates of the floR-T1 gene in the $P$. aeruginosa strains collected in $2008-2009$ and $2015-2017$ were $3.00 \%(6 / 200)$ and $7.39 \%(17 / 230)$, respectively. FloR-T2 exhibited less identity with other FloR proteins than FloRv. The two floR variants, floR-T1 and floR-T2, in P. aeruginosa TL1285 were functionally active and located on a novel incomplete Tn 4371 family integrative and conjugative element (ICE). The expression of the two floR variants could be induced by florfenicol and chloramphenicol at different levels.

Conclusions: Two floR variants, floR-T1 and floR-T2, were identified in a clinical $P$. aeruginosa strain. Tn 4371 family ICEs contribute to the dissemination of resistance genes among $P$. aeruginosa. Antimicrobial resistance could be transmitted from animal bacteria to human pathogens, posing a severe threat to public health.

\section{Background}

Florfenicol, a fluorinated thiamphenicol derivative, has been licensed in China since 1999 and has been widely used to control respiratory tract diseases and enteric infections in food-producing animals [1]. However, due to the inappropriate use of florfenicol to prevent or cure bacterial infections, florfenicol resistance has become increasingly serious in veterinary medicine [2]. Although florfenicol is not approved for use in humans, an increasing number of studies have reported dramatic and serious florfenicol resistance in various clinical strains, such as Pasteurella multocida, Salmonella, and Klebsiella pneumoniae [3-5].

To date, seven florfenicol resistance genes (excluding variants), floR, fexA, fexB, cfr, optrA, pexA and estDL 136, have been reported; [6-13] among them, floR is one of the main florfenicol resistance genes in gram-negative bacteria [14]. Several variants of the floR gene, including $p p$-flo, cm/A-like, floRv and floSt, have been documented, and most of them encode 404 amino acids. These floR variants are closely related to each other, and floRv from Stenotrophomonas maltophilia shares the lowest amino acid 
identity (88.4\%-91.8\%) with the others excluding pp-flo [14]. The floR gene has been detected either on chromosomes or plasmids of various bacteria and has often been associated with mobile genetic elements and genomic islands.

As an opportunistic pathogen, Pseudomonas aeruginosa is a major cause of nosocomial infections, such as respiratory infections in debilitated patients and patients with cystic fibrosis, and is known for its resistance to antibiotics $[15,16]$. Typically, $P$. aeruginosa is clinically resistant to chloramphenicol, but rifampicin-tobramycin conjugates have been shown to break the intrinsic resistance of $P$. aeruginosa to chloramphenicol in vitro or in vivo, thus expanding the therapeutic usefulness of this agent [17]. However, the $f l o R$ gene carried by this pathogen may cause this strategy to fail when chloramphenicol is used. In this study, we screened the floR gene of clinical $P$. aeruginosa isolates collected in the years 2008-2009 and 2015-2018. As a result, a $P$. aeruginosa strain named TL1285 carrying two functionally active floR variants was identified.

\section{Methods}

\section{Bacterial isolation and sequencing}

A total of 430 clinical $P$. aeruginosa strains isolated from sputum, urine or blood samples of patients were collected at the First Affiliated Hospital of Wenzhou Medical University, Wenzhou, China. Among these isolates, approximately 200 strains were isolated from 2008-2009, and 230 strains were isolated from 2015-2017. Each purified isolate was incubated overnight in $5 \mathrm{ml}$ of Luria-Bertani (LB) broth at $37^{\circ} \mathrm{C}$ for 16 hours, and genomic DNA was extracted using an AxyPrep Bacterial Genomic DNA Miniprep kit (Axygen Scientific, Union City, CA, USA). According to the isolated periods, the genomic DNA was pooled as TL0809 and TL151617 and subsequently used for high-throughput sequencing by the lllumina pairedend strategy. Genomic DNA of TL1285 was also sequenced by Pacific Bioscience sequencers at Annoroad Gene Technology Co., Ltd. (Beijing, China).

\section{Genome assembly, annotation, and bioinformatics analysis}

Genome assembly of pooled DNA sequencing was performed using megahit [18], and contigs less than $400 \mathrm{bp}$ were discarded. The complete genome of $P$. aeruginosa TL1285 was assembled using Canu [19] with long reads obtained from PacBio. Error correction of tentative complete circular sequence was performed using Pilon [20] with short read sets derived from Illumina sequencing. Open reading frames (ORFs) of pooled DNA sequences were predicted using Prodigal [21] with default parameters. Using the antibiotic resistance genes of the CARD [22] and ResFinder [23] databases as a query, a BLASTN search was performed against the two assembled sequences of the pooled DNA with thresholds of $>70 \%$ nucleotide identity and $>80 \%$ alignment coverage. Gene prediction and annotation of TL1285 were initially performed with RAST [24] and then verified by BLASTP searches against the UniProtKB/SwissProt [25] and RefSeq [26] databases. Annotation of mobile genetic elements was carried out using online databases including ISfinder [27], INTEGRALL [28], and the Tn Number Registry [29]. 


\section{PCR amplification and cloning of the floR gene}

Genomic DNA of each of the 430 isolates was screened for the floR gene using a PCR method with the primers listed in Table S1. PCR amplification was carried out under the following conditions: initial denaturation of $5 \mathrm{~min}$ at $95^{\circ} \mathrm{C} ; 35$ cycles of denaturation $\left(94^{\circ} \mathrm{C}\right.$ for $45 \mathrm{~s}$ ), annealing $\left(58^{\circ} \mathrm{C}\right.$ for $45 \mathrm{~s}$ ), and extension $\left(72{ }^{\circ} \mathrm{C}\right.$ for $\left.90 \mathrm{~s}\right)$; and a final extension step at $72{ }^{\circ} \mathrm{C}$ for $10 \mathrm{~min}$. The floR-T1 and floR-T2 gene sequences with promoter regions were amplified from $P$. aeruginosa TL1285 and ligated into pUCP24, and the recombinant plasmids were transformed into $P$. aeruginosa PA01.

\section{Antimicrobial susceptibility testing}

Minimum inhibitory concentrations (MICs) of 17 antimicrobial agents were determined using the agar dilution method and interpreted in accordance with the guidelines of the Clinical and Laboratory Standards Institute (CLSI document M100-S27, 2017).

\section{Comparison of the expression of floR-T1 and floR-T2 in P. aeruginosa TL1285}

Quantitative reverse transcription PCR (qRT-PCR) was used to investigate the expression of the floR variants in TL1285 in the presence/absence of $2 \mathrm{mg} / \mathrm{L}$ florfenicol or chloramphenicol. In brief, RNA was extracted from $3 \mathrm{~mL}$ of LB broth culture $(\mathrm{OD} 600=1)$ of $P$. aeruginosa TL1285 using TRIzol Reagent (Invitrogen, USA) following the manufacturer's instructions. RNA $(1 \mu \mathrm{g})$ was used as the template for cDNA synthesis using HiScript II Reverse Transcriptase (Vazyme, Nanjing, China) following the manufacturer's instructions. qRT-PCR was used to quantify the amount of floR-T1 and floR-T2 in CDNAs using ChamQ Universal SYBR qPCR Master Mix (Vazyme, Nanjing, China) following the manufacturer's instructions with qPCR primers (Table S1).

\section{Results And Discussion}

\section{Identification of the floR variants}

To investigate the prevalence of the floR gene among clinical $P$. aeruginosa isolates, bacterial genomic DNA was pooled according to the isolated periods as TL0809 (200 P. aeruginosa strains isolated from 2008-2009) and TL151617 (230 P. aeruginosa strains isolated from 2015-2017). The Illumina sequencing of TL0809 and TL151617 generated $1.4 \times 10^{8}$ and $4.6 \times 10^{8}$ filtered reads with average depths of $\sim 20$ and $\sim 50$, respectively. The reads of TL0809 and TL151617 were assembled to generate 20,205 and 41,932 contigs, with N50 values of 3,550 and 2,316 bp in length, respectively. Using the floR gene

(AF231986) as a reference, three types of floR variants (the reference floR, floR-T1 and floR-T2, named in this study) were identified in the two pooled sequencing libraries, of which TL0809 contained all three and TL151617 contained only two floR variants (the reference floR and floR-T1) (Table 1). 
Table 1.

The Abundance and PCR positive rate of the floR variants

\begin{tabular}{|clllll|}
\hline & floR variants & Identity & Match length (aa) & Abundance $^{\mathrm{b}}$ & PCR positive rate $^{-}$ \\
\hline TL050607 & floR & $99.3 \%$ & 404 & 0.68 & $2 / 200(1.00 \%)$ \\
& floR-T1 & $91.3 \%$ & 404 & 1.18 & $6 / 200(3.00 \%)$ \\
\hline floR-T2 & $87.6 \%$ & 404 & 0.32 & $1 / 200(0.50 \%)$ \\
\hline floR & $99.3 \%$ & 404 & 0.56 & $3 / 230(1.30 \%)$ \\
\hline & floR-T1 & $91.3 \%$ & 404 & 4.95 & $17 / 230(7.39 \%)$ \\
\hline
\end{tabular}

a reference floR (AF231986)

${ }^{\mathrm{b}}$ Abundance $=$ average fold of gene $* 4 * 100 /$ average fold of $16 \mathrm{~S}$ rRNA

Using homology tree analysis, the amino acid identities of FloR-T1 and FloR-T2 with the known floR proteins ranged from $90.80-100 \%$ and $86.10-88.90 \%$, respectively (Fig. S1). FloR-T1 was identical to the FloR protein (YP_001715371.1) identified in Acinetobacter baumannii, while FloR-T2 showed the highest identity (88.90\%) with the FloR protein (YP_005351917.1) identified in Klebsiella pneumoniae. FloRv was the floR variant with the lowest identity $(88.40 \%-91.80 \%)$ to other FloR proteins reported so far. However, FloR-T2 exhibited less identity with other FloR proteins than FloRv. Furthermore, FloR-T2 was shown to be one of the most divergent members of the FloR family, followed by FloRv (Fig. S1). Therefore, floR-T2 is a novel floR variant identified in this study.

The result of PCR amplification of the reference $f l o R, f l o R-T 1$ and floR-T2 genes showed that the most prevalent variant was floR-T1, while floR-T2 was only identified in the isolates collected from 2008-2009 (Table 1). The positive rates were consistent with the abundance of the corresponding genes in the pooled genomic DNA sequencing libraries. The positive rate of the floR-T1 gene in the strains collected from $2015-2017(7.39 \%, 17 / 230)$ was increased compared with that from $2008-2009(3.00 \%, 6 / 200)$, which was also similar to the positive rate of the floR gene $(7.01 \%, 23 / 328)$ of the human clinical $K$. pneumoniae isolates collected from the same district [5]. Zhan et al. also reported a dramatic increase in florfenicol-resistant invasive nontyphoidal Salmonella (iNTS) in China between 2007 and 2016 [3]. The results indicate that antimicrobial resistance could be transmitted from animal bacteria to human pathogens, which might pose a severe threat to public health.

\section{Antimicrobial susceptibility of $P$. aeruginosa TL1285 and the floR clone}

Among all $P$. aeruginosa strains, only one strain named TL1285, isolated from a sputum sample in 2008, carried both floR-T1 and floR-T2. P. aeruginosa TL1285 was resistant to both chloramphenicol and florfenicol (Table 2). Cloned floR-T1 (pUCP24-floR-T1/PA01) increased the MICs for chloramphenicol and florfenicol by 2- and 4-fold, and the MICs of chloramphenicol and florfenicol against the transformant 
carrying floR-T2 (pUCP24-floR-T2/PA01) increased 4- and 8-fold, respectively. The results indicate that floR-T1 and floR-T2 of $P$. aeruginosa TL1285 are functionally active.

Table 2

Antimicrobial susceptibility of $P$. aeruginosa TL1285

\begin{tabular}{|ll|}
\hline Antibiotics & MIC $(\mu \mathrm{g} / \mathrm{mL})$ \\
\hline Ampicillin & 1024 \\
\hline Ceftazidime & $<1$ \\
\hline Levofloxacin & $<0.5$ \\
\hline Cefpyridine & 4 \\
\hline Minocycline & 64 \\
\hline Chloramphenicol & 128 \\
\hline Florfenicol & 256 \\
\hline Ciprofloxacin & 2 \\
\hline Azithromycin & 32 \\
\hline Fosfomycin & 256 \\
\hline Tigecycline & 4 \\
\hline Colistin & $<1$ \\
\hline Erythromycin & 256 \\
\hline Nalidixic acid & $>1024$ \\
\hline Gentamicin & $>1024$ \\
\hline Kanamycin & 64 \\
\hline Streptomycin & $>1024$ \\
\hline
\end{tabular}

\section{Expression of the floR variants}

Using transcriptome sequencing, Lang et al. found that the expression of the floR gene of the $E$. coli plasmid pAR060302 increased 8-fold under the induction of florfenicol [30]. Yinghui et alalso reported that the mRNA levels of the floR gene encoded by ICEApI2 on chromosomes increased in the presence of chloramphenicol [31]. In this work, we performed qRT-PCR to explore the expression of the two floR variants with or without florfenicol and chloramphenicol induction. The mRNA levels of floRT2 in P. aeruginosa TL1285 increased 78-fold and 56-fold in the presence of florfenicol and 
chloramphenicol, while the mRNA levels of floR-T1 only increased 1.2-fold and 1.6-fold in the presence of florfenicol and chloramphenicol, respectively (Fig. 1).

It is known that the expression of chloramphenicol resistance genes, including $\operatorname{cat} A, \operatorname{cm} I A$ and fexA, could be induced by chloramphenicol, and this induction is mediated by a translational attenuator at the posttranscriptional level $[8,13,32]$. The translational attenuators that located immediately upstream of these chloramphenicol resistance genes consist of a single pair of inverted repeated sequences, IR1 and IR2, as well as a reading frame of a 6-9 aa short peptide [13]. IR1 and IR2 were able to form a stable stem-loop structure blocking the resistance gene-associated ribosome binding site (RBS). Yinghui et al reported that the region upstream of the floR genes also contains a well-conserved similar attenuator sequence [31]. However, the stable stem-loop structure of the attenuator sequence did not overlap with the RBS site of the floR gene. Interestingly, we found that the peptide sequence of the floR-associated attenuator sequence in pA060302 and ICEApl2 reported by Yinghui [31] was identical to that of floR-T1 but different from that of floRv or floR-T2 by 3 amino acids (Fig. 2). Furthermore, the attenuator sequences of floR$T 2$ and $f l o R v$ encode an identical peptide sequence, although their nucleotide sequence varies by one base. The inverted repeats of the attenuators of these floR genes were also different, and the stem-loop structures formed in distinct stable states. The genes floR-T2 and floRv have the most stable structure among the four variants. Considering that the stem-loop structure is distant to the RBS site of the floR gene, it is not clear whether this structure participates in the induced expression of the floR gene.

\section{floR-T2 encoded in a Tn4371-like ICE}

Whole genome sequencing (WGS) of $P$. aeruginosa TL1285 only produced a circular 6,609,407 bp chromosome with an average GC content of $66.06 \%$ encoding 5,611 ORFs. Multiple antimicrobial resistance genes, including resistance genes for $\beta$-lactams $\left(b / a_{\mathrm{OXA}-50}\right.$ and $\left.b / a_{\mathrm{PDC}-3}\right)$, aminoglycosides (aadA5 and $\operatorname{aac}(3)-I / a)$, sulfonamides (sul1), tetracycline (tetG), chloramphenicol (catB7, floR-T1 and floRT2) and fosfomycin ( $f o s A$ ), were identified in the $P$. aeruginosa TL1285 genome. The antimicrobial resistance phenotype was in accordance with the genotype (Table 2). The florfenicol-resistant genes floR$T 1$ and floR-T2 were embedded in an 86-kb Tn4371-like integrative and conjugative element (ICE) (Fig. 3). Tn 4371 is a 55-kb ICE that can be integrated into the attB site (5'-TTTTCAT-3') through a site-specific recombination process since the ends of the element can be detected covalently as a transfer intermediate [33, 34].

To track the epidemiological correlation between floR-T2 and genome islands, a BLASTN search was performed against the GenBank database using floR-T2 as a query. A total of five $P$. aeruginosa chromosomes, WPB099 (CP031878), WPB100 (CP031877), WPB101 (CP031876), PASGNDM345 (CP020703) and PASGNDM699 (CP020704), and one E. cloacae chromosome, AR_038 (CP030347), were found to carry floR-T2. MLST analysis of the five $P$. aeruginosa chromosomes together with $P$. aeruginosa TL1285 showed that WPB099, WPB100, WPB101, PASGNDM345 and PASGNDM699 belonged to ST308, while TL1285 belonged to ST316. P. aeruginosa ST308 is a high-risk clone that can locally acquire resistance determinants originated from the water-distribution system and caused a five-year outbreak in 
patients in a French hospital [35]. Interestingly, these floR-T2-carrying strains came from different resources. P. aeruginosa WPB099, WPB100 and WPB101 were isolated from hospital wastewaters in Singapore. $P$. aeruginosa PASGNDM345 and PASGNDM699 were derived from patient sputum in Singapore, while E. cloacae AR_038 and TL1285 were isolated from patient sputum in the United States and China, respectively. Whole genome alignment of six $P$. aeruginosa strains suggested that the strains shared high identity, and their differences were mainly focused on some genomic islands (Fig. 3). The Tn 4371-like ICE carrying floR-T2 in TL1285 was also partially present in these five $P$. aeruginosa strains. It may be noted that WPB099, WPB100 and WPB101 were not fully sequenced, and the floR-T2 gene was located on an approximately $10 \mathrm{~kb}$ separate segment, so the precise genetic environments around floR-T2 could not be described.

Tn4371-like ICEs are mosaic in structure and consist of Ti-RP4-like transfer systems, an integrase region, plasmid maintenance genes and accessory genes [34]. Comparative analysis of the Tn4371-like ICE regions of six $P$. aeruginosa strains revealed that the plasmid maintenance system (repA, parA and parB) and conjugational transfer systems were conserved among these sequences (Fig. 4). The variable region between the traF and traR genes, which encoded a biphenyl catabolic bph gene cluster in Tn 4371 (AJ536756), was different in these six P. aeruginosa isolates. The variable regions of WPB099, WPB100 and WPB101 were a 20-kb fragment encoding the oqxB32 gene, which confers resistance to quinolone. The variable regions of PASGNDM345 and PASGNDM699 shared high identity with those of WPB099, WPB100 and WPB101. The only difference was that in PASGNDM345 and PASGNDM699, a 13.7-kb fragment was inserted between $c z c D$ and $l y s R$, which encode $b / a_{\mathrm{NDM}-1}, \operatorname{msr}(E)$ and $f l o R-T 2$ genes. The insertion fragment was flanked by 695 bp direct repeats, which were also present in WPB099, WPB100 and WPB101, whereas it showed no similarity to the existing IS element [36]. The variable region of TL1285 was similar to those of PASGNDM345 and PASGNDM699, except that the b/a $a_{\mathrm{NDM}-1}-h p-m s r(E)$ genes of PASGNDM345 and PASGNDM699 were replaced by floR-T1-tetR-tetA-lysR in TL1285. The results indicate that Tn4371-like ICEs might have emerged as a potential vehicle to mediate the spread of drug resistance genes in $P$. aeruginosa isolates.

Any ICE that encodes an integrase gene closely related to int $_{\mathrm{Tn} 4371}(>70 \%$ protein homology) and has similar maintenance and transfer genes could be considered a member of the Tn 4371 family [37]. The integrase genes (int) of PASGNDM345, PASGNDM699, WPB99, WPB100 and WPB101 were identical and shared $78 \%$ identity with that of Tn4371. However, no homologue of $i n t_{\mathrm{Tn} 4371}$ was found in TL1285 (Fig. 4). Tn 4371 family ICEs could be integrated into the genome through an 8-bp attB site, generating direct repeat attL and attR element chromosomal junctions [33]. In PASGNDM345, PASGNDM699, WPB99, WPB100 and WPB101, 8-bp repeats (5'-TTTTTTGT-3') were identified on both extremities of the ICE region. However, in TL1285, only attR was found (Fig. 4). The noc gene upstream of repA in TL1285 was truncated by a novel Tn 402 family transposon. The transposon is formed by ISCfr1 and In 2 carrying a single aadA5 cassette embedded downstream of the tnpR gene of Tn 1013, and this Tn 402 family transposon was surrounded by 37-bp imperfect inverted repeats (Fig. S2). 
Inverse PCR using primers P1, P2 and P3 (Fig. 4) was performed to detect whether the ICE in TL1285 could generate a circular extrachromosomal form, but no positive results were observed. Taken together, we speculate that the ICE in TL1285 is an incomplete member of the Tn4371 family and may have lost the ability to excise and integrate. The insertion of the Tn 402 family transposon leads to the loss of the upstream sequence of the nor gene, including the integrase gene int of ICE.

\section{Conclusion}

In conclusion, three floR variants, floR, floR-T1 and floR-T2, were identified in clinical $P$. aeruginosa, and floR-T1 was the most prevalent variant. The gene floR-T2 was a novel floR variant identified in this study that showed less identity with other FloR proteins than FloRv. One $P$. aeruginosa strain, TL1285, carrying floR-T1 and floR-T2 was identified, and the whole genome of this strain was sequenced. The floR-T2 gene of TL1285 was located on an incomplete novel Tn 4371 family ICE, and similar ICEs were also identified in five other floR-T2-carrying $P$. aeruginosa genomes. The expression of the two floR variants could be induced by florfenicol and chloramphenicol at different levels. Our results indicate that Tn 4371 family ICEs may cause the dissemination of floR-T2 and other drug resistance genes in $P$. aeruginosa. Antimicrobial resistance could be transmitted from animal bacteria to human pathogens, posing a severe threat to public health.

\section{Abbreviations}

ICE, Integrative and conjugative element; BLAST, The Basic Local Alignment Search Tool; MIC, Minimum Inhibitory Concentration; qRT-PCR, Quantitative reverse transcription PCR; ORFs, Open reading frames; RBS, ribosome binding site; PCR: polymerase chain reaction. WGS, Whole genome sequencing.

\section{Declarations}

\section{Acknowledgments}

The authors would like to acknowledge all study participants and individual who contributed for the study.

\section{Authors' contributions}

JL, HX, HYL, XL, KL and HZ collected the strains. HML, WL, JC, XYZ, KS and QL performed the experiments. QC, HLL and $X Z$ analyzed the experimental results. $C Q, W Z$ and $A L$ performed the bioinformatics analysis. CQ, TX and QB co-led the writing of the manuscript. CQ and QB designed the work. All authors read and approved the final manuscript

\section{Funding}


This study was supported by the Science \& Technology Project of Wenzhou City, China [Y20170205 and 2019Y0358]; Science and Technology Project of Lishui City, China [2017GYX07]; the National Natural Science Foundation of China [81973382, 81960381]; the Natural Science Foundation of Zhejiang Province, China [LQ17H190001 and LY19C060002] and the Science \& Technology Project of Inner Mongolia Autonomous Region, China.

\section{Availability of data and materials}

All data generated or analyzed during this study are included in this published article and its supplementary information files. The data related to the paper are deposited in the NCBI GenBank. The accession numbers for $P$. aeruginosa TL1285 chromosome is CP053390.

\section{Ethics approval and consent to participate}

Not applicable.

\section{Consent for publication}

Not applicable.

\section{Competing interests}

The authors have no competing interests to declare.

\section{References}

1. Zhao Q, Wang $Y$, Wang S, Wang Z, Du XD, Jiang H, et al. Prevalence and Abundance of Florfenicol and Linezolid Resistance Genes in Soils Adjacent to Swine Feedlots. Sci Rep. 2016;6:32192; doi:10.1038/srep32192

2. Chang S-K, Lo D-Y, Wei H-W, Kuo H-C. Antimicrobial resistance of Escherichia coli isolates from canine urinary tract infections. Journal of Veterinary Medical Science. 2014:13-0281; doi:10.1292/jvms.13-0281

3. Zhan Z, Xu X, Shen H, Gao Y, Zeng F, Qu X, et al. Rapid Emergence of Florfenicol-Resistant Invasive Non-Typhoidal Salmonella in China: A Potential Threat to Public Health. The American journal of tropical medicine and hygiene. 2019;101(6):1282-5; doi:10.4269/ajtmh.19-0403

4. Ujvari B, Weiczner R, Deim Z, Terhes G, Urban E, Toth AR, et al. Characterization of Pasteurella multocida strains isolated from human infections. Comparative immunology, microbiology and infectious diseases. 2019;63:37-43; doi:10.1016/j.cimid.2018.12.008 
5. Lu J, Zhang J, Xu L, Liu Y, Li P, Zhu T, et al. Spread of the florfenicol resistance floR gene among clinical Klebsiella pneumoniae isolates in China. Antimicrobial resistance and infection control. 2018;7:127; doi:10.1186/s13756-018-0415-0

6. Arcangioli MA, Leroy-Sétrin S, Martel JL, Chaslus-Dancla E. A new chloramphenicol and florfenicol resistance gene flanked by two integron structures in Salmonella typhimurium DT104. FEMS microbiology letters. 1999;174(2):327-32; doi:10.1111/j.1574-6968.1999.tb13586.x

7. Schwarz S, Werckenthin C, Kehrenberg C. Identification of a plasmid-borne chloramphenicolflorfenicol resistance gene in Staphylococcus sciuri. Antimicrobial agents and chemotherapy. 2000;44(9):2530-3; doi:10.1128/AAC.44.9.2530-2533.2000

8. Kehrenberg C, Schwarz S. fexA, a novel Staphylococcus lentus gene encoding resistance to florfenicol and chloramphenicol. Antimicrobial agents and chemotherapy. 2004;48(2):615-8; doi:10.1128/AAC.48.2.615-618.2004

9. Lang KS, Anderson JM, Schwarz S, Williamson L, Handelsman J, Singer RS. Novel florfenicol and chloramphenicol resistance gene discovered in Alaskan soil by using functional metagenomics. Applied and environmental microbiology. 2010;76(15):5321-6; doi:10.1128/AEM.00323-10

10. Liu H, Wang Y, Wu C, Schwarz S, Shen Z, Jeon B, et al. A novel phenicol exporter gene, fexB, found in enterococci of animal origin. J Antimicrob Chemother. 2012;67(2):322-5; doi:10.1093/jac/dkr481

11. Tao W, Lee MH, Wu J, Kim NH, Kim J-C, Chung E, et al. Inactivation of chloramphenicol and florfenicol by a novel chloramphenicol hydrolase. Applied and environmental microbiology. 2012;78(17):6295-301; doi:10.1128/AEM.01154-12

12. Wang Y, Lv Y, Cai J, Schwarz S, Cui L, Hu Z, et al. A novel gene, optrA, that confers transferable resistance to oxazolidinones and phenicols and its presence in Enterococcus faecalis and Enterococcus faecium of human and animal origin. J Antimicrob Chemother. 2015;70(8):2182-90; doi:10.1093/jac/dkv116

13. Schwarz S, Kehrenberg C, Doublet B, Cloeckaert A. Molecular basis of bacterial resistance to chloramphenicol and florfenicol. FEMS Microbiol Rev. 2004;28(5):519-42; doi:10.1016/j.femsre.2004.04.001

14. He T, Shen J, Schwarz S, Wu C, Wang Y. Characterization of a genomic island in Stenotrophomonas maltophilia that carries a novel floR gene variant. J Antimicrob Chemother. 2015;70(4):1031-6; doi:10.1093/jac/dku491

15. Breidenstein EBM, de la Fuente-Núñez C, Hancock REW. Pseudomonas aeruginosa: all roads lead to resistance. Trends Microbiol. 2011;19(8):419-26; doi:10.1016/j.tim.2011.04.005

16. Domalaon R, Idowu T, Zhanel GG, Schweizer F. Antibiotic Hybrids: the Next Generation of Agents and Adjuvants against Gram-Negative Pathogens? Clinical microbiology reviews. 2018;31(2); doi:10.1128/CMR.00077-17

17. Idowu T, Arthur G, Zhanel GG, Schweizer F. Heterodimeric Rifampicin-Tobramycin conjugates break intrinsic resistance of Pseudomonas aeruginosa to doxycycline and chloramphenicol in vitro and in 
a Galleria mellonella in vivo model. Eur J Med Chem. 2019;174:16-32;

doi:10.1016/j.ejmech.2019.04.034

18. Li D, Liu CM, Luo R, Kunihiko S, Tak-Wah LJB. MEGAHIT: an ultra-fast single-node solution for large and complex metagenomics assembly via succinct de Bruijn graph. Bioinformatics. 2015(10):10; doi:10.1093/bioinformatics/btv033

19. Koren S, Walenz BP, Berlin K, Miller JR, Bergman NH, Phillippy AM. Canu: scalable and accurate longread assembly via adaptive k-mer weighting and repeat separation. Genome Res. 2017;27(5):722-36; doi:10.1101/gr.215087.116

20. Walker BJ, Abeel T, Shea T, Priest M, Abouelliel A, Sakthikumar S, et al. Pilon: an integrated tool for comprehensive microbial variant detection and genome assembly improvement. PloS one. 2014;9(11):e112963; doi:10.1371/journal.pone.0112963

21. Hyatt D, Chen G-L, LoCascio PF, Land ML, Larimer FW, Hauser LJ. Prodigal: prokaryotic gene recognition and translation initiation site identification. BMC Bioinformatics. 2010;11(1):119-0; doi:10.1186/1471-2105-11-119

22. Jia B, Raphenya AR, Alcock B, Waglechner N, Guo P, Tsang KK, et al. CARD 2017: expansion and model-centric curation of the comprehensive antibiotic resistance database. Nucleic Acids Res. 2017;45(D1):D566-D73; doi:10.1093/nar/gkw1004

23. Ea Z, Henrik H, Salvatore C, Martin V, Simon R, Ole L, et al. Identification of acquired antimicrobial resistance genes. J Antimicrob Chemother. 2012;67(11):11; doi:10.1093/jac/dks261

24. Aziz RK, Bartels D, Best AA, DeJongh M, Disz T, Edwards RA, et al. The RAST Server: Rapid Annotations using Subsystems Technology. BMC genomics. 2008;9(1):75-0; doi:10.1186/1471-21649-75

25. Boutet E, Lieberherr D, Tognolli M, Schneider M, Bansal P, Bridge AJ, et al. UniProtKB/Swiss-Prot, the Manually Annotated Section of the UniProt KnowledgeBase: How to Use the Entry View. Methods Mol Biol. 2016;1374:23-54; doi:10.1007/978-1-4939-3167-5_2

26. O'Leary NA, Wright MW, Brister JR, Ciufo S, Haddad D, McVeigh R, et al. Reference sequence (RefSeq) database at NCBI: current status, taxonomic expansion, and functional annotation. Nucleic Acids Res. 2016;44(D1):D733-45; doi:10.1093/nar/gkv1189

27. Siguier P, Perochon J, Lestrade L, Mahillon J, Chandler M. ISfinder: the reference centre for bacterial insertion sequences. Nucleic Acids Res. 2006;34(Database issue):D32-6; doi:10.1093/nar/gkj014

28. Moura A, Soares M, Pereira C, Leitao N, Henriques I, Correia A. INTEGRALL: a database and search engine for integrons, integrases and gene cassettes. Bioinformatics. 2009;25(8):1096-8; doi:10.1093/bioinformatics/btp105

29. Roberts AP, Chandler M, Courvalin P, Guedon G, Mullany P, Pembroke T, et al. Revised nomenclature for transposable genetic elements. Plasmid. 2008;60(3):167-73; doi:10.1016/j.plasmid.2008.08.001

30. Lang KS, Danzeisen JL, Xu W, Johnson TJJA, Microbiology E. Transcriptome Mapping of pAR060302, a blaCMY-2-Positive Broad-Host-Range IncA/C Plasmid. Appl Environ Microbiol. 2012;78(9):3379-86; doi:10.1128/AEM.07199-11 
31. Li Y, Li Y, Fernandez Crespo R, Leanse LG, Langford PR, Bosse JT. Characterization of the Actinobacillus pleuropneumoniae SXT-related integrative and conjugative element ICEApl2 and analysis of the encoded FloR protein: hydrophobic residues in transmembrane domains contribute dynamically to florfenicol and chloramphenicol efflux. J Antimicrob Chemother. 2018;73(1):57-65; doi:10.1093/jac/dkx342

32. Stokes H, Hall R. Sequence Analysis of the Inducible Chloramphenicol Resistance Determinant in the Tn1696 Integron Suggests Regulation. Plasmid. 1991;26:10-9; doi:10.1016/0147-619X(91)90032-R

33. Merlin C, Springael D, Toussaint A. Tn4371: A modular structure encoding a phage-like integrase, a Pseudomonas-like catabolic pathway, and RP4/Ti-like transfer functions. Plasmid. 1999;41(1):40-54; doi:10.1006/plas.1998.1375

34. Toussaint A, Merlin C, Monchy S, Benotmane MA, Leplae R, Mergeay M, et al. The biphenyl- and 4chlorobiphenyl-catabolic transposon Tn4371, a member of a new family of genomic islands related to IncP and Ti plasmids. Appl Environ Microbiol. 2003;69(8):4837-45; doi:10.1128/aem.69.8.48374845.2003

35. Jeanvoine A, Meunier A, Puja H, Bertrand X, Valot B, Hocquet D. Contamination of a hospital plumbing system by persister cells of a copper-tolerant high-risk clone of Pseudomonas aeruginosa. Water Res. 2019;157:579-86; doi:10.1016/j.watres.2019.04.011

36. Ding Y, Teo JWP, Drautz-Moses DI, Schuster SC, Givskov M, Yang L. Acquisition of resistance to carbapenem and macrolide-mediated quorum sensing inhibition by Pseudomonas aeruginosa via ICETn4371 6385. Communications biology. 2018;1:57; doi:10.1038/s42003-018-0064-0

37. Ryan MP, Pembroke JT, Adley CC. Novel Tn4371-ICE like element in Ralstonia pickettii and genome mining for comparative elements. BMC Microbiol. 2009;9:242; doi:10.1186/1471-2180-9-242

\section{Figures}




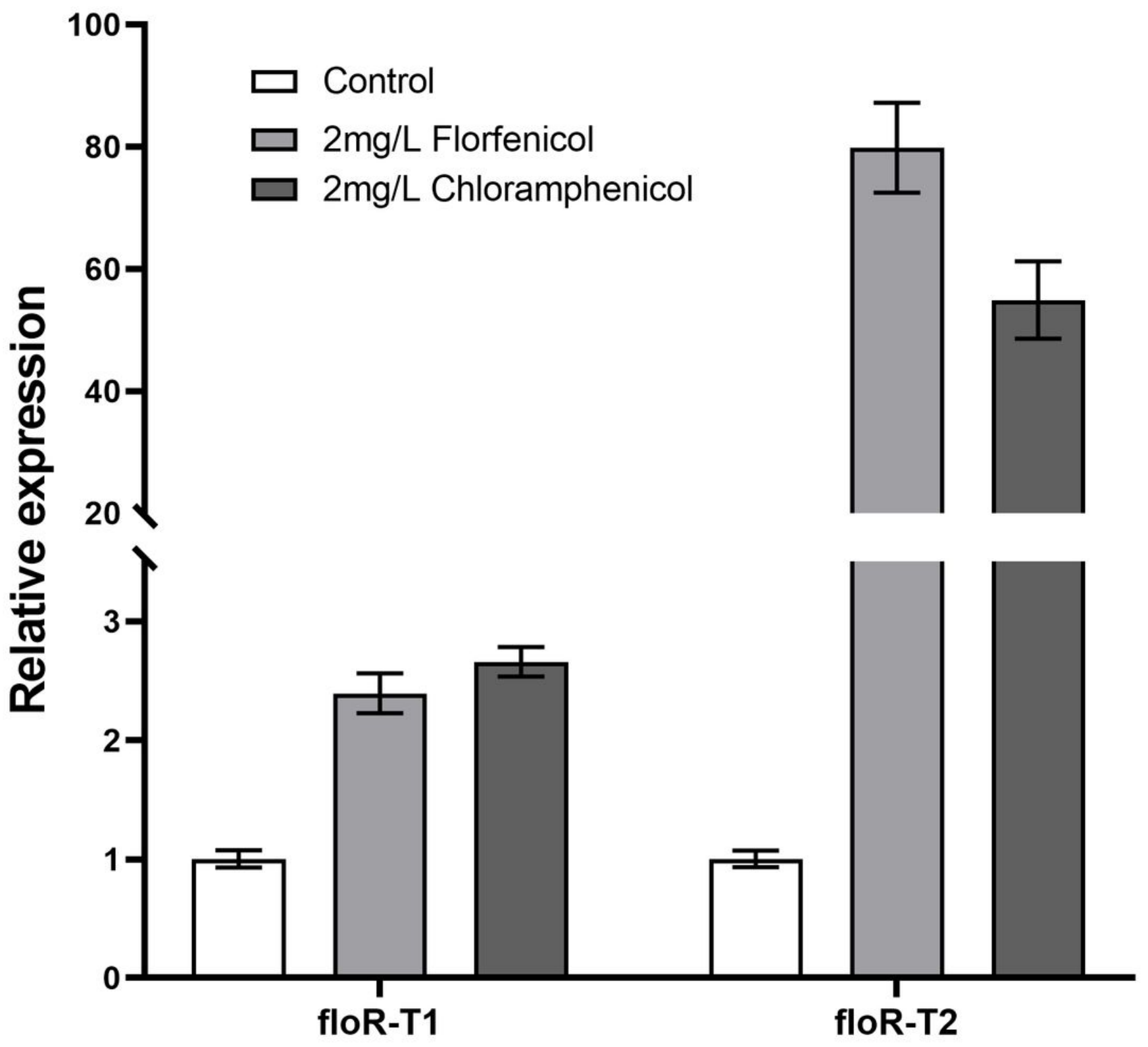

Figure 1

Expression of the floR variants in TL1285 with or without florfenicol or chloramphenicol induction. 
(a)

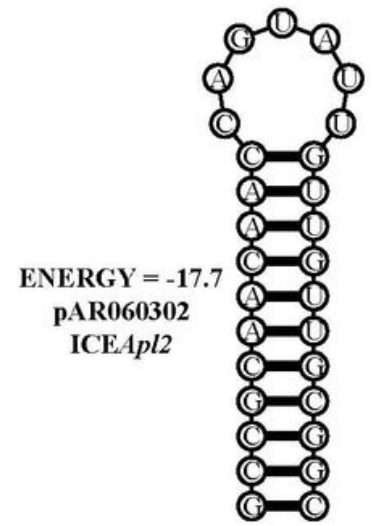

(b)

pAR060302 ICE Apl2 floRv floR-T1 floR-T2

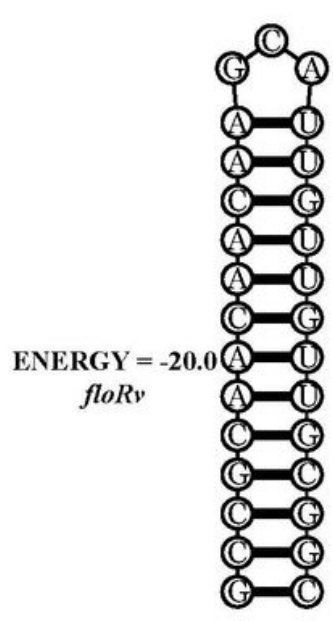

ENERGY = -16.0 floR-T1

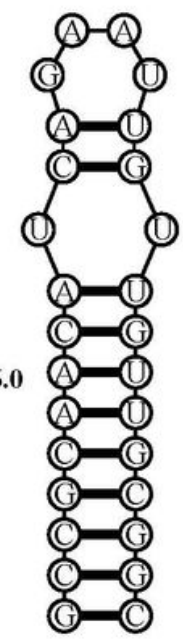

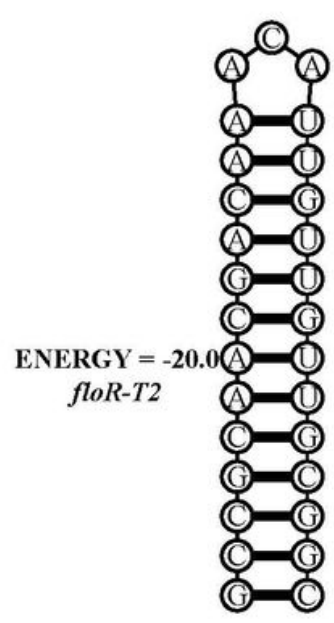

stem-loop region

peptide encoding region

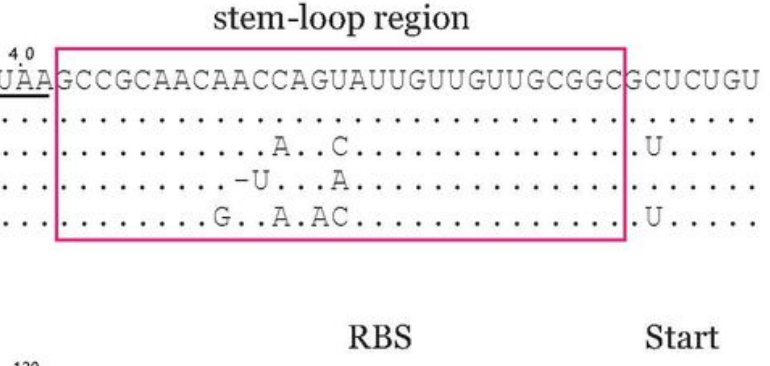

- MGASTTADK

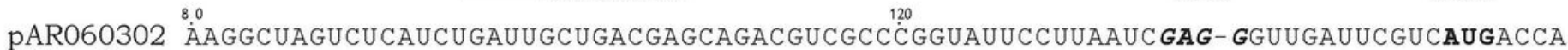

RBS

Start ICEApl2

floRv

floR-T1

floR-T2

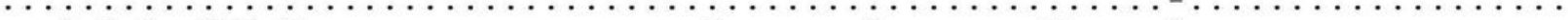

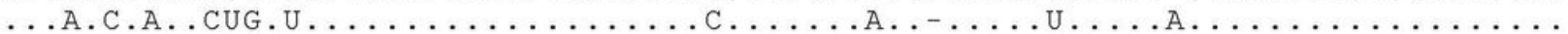

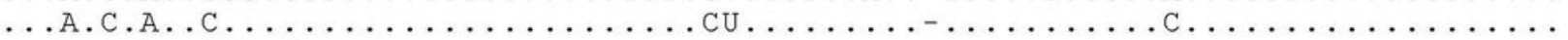

..A.C.A.CUU.U.

\section{Figure 2}

Comparison of sequences upstream of the floR variants. (a) The stable mRNA secondary structure of floR variants formed by inverted repeat sequences boxed in (b). (b) The attenuator of floR variants consists of a peptide encoding region (bold type) and stem-loop region (boxed). The start codons and ribosome binding sites (RBS) of short peptide and floR are labeled and displayed in bold type letters. 


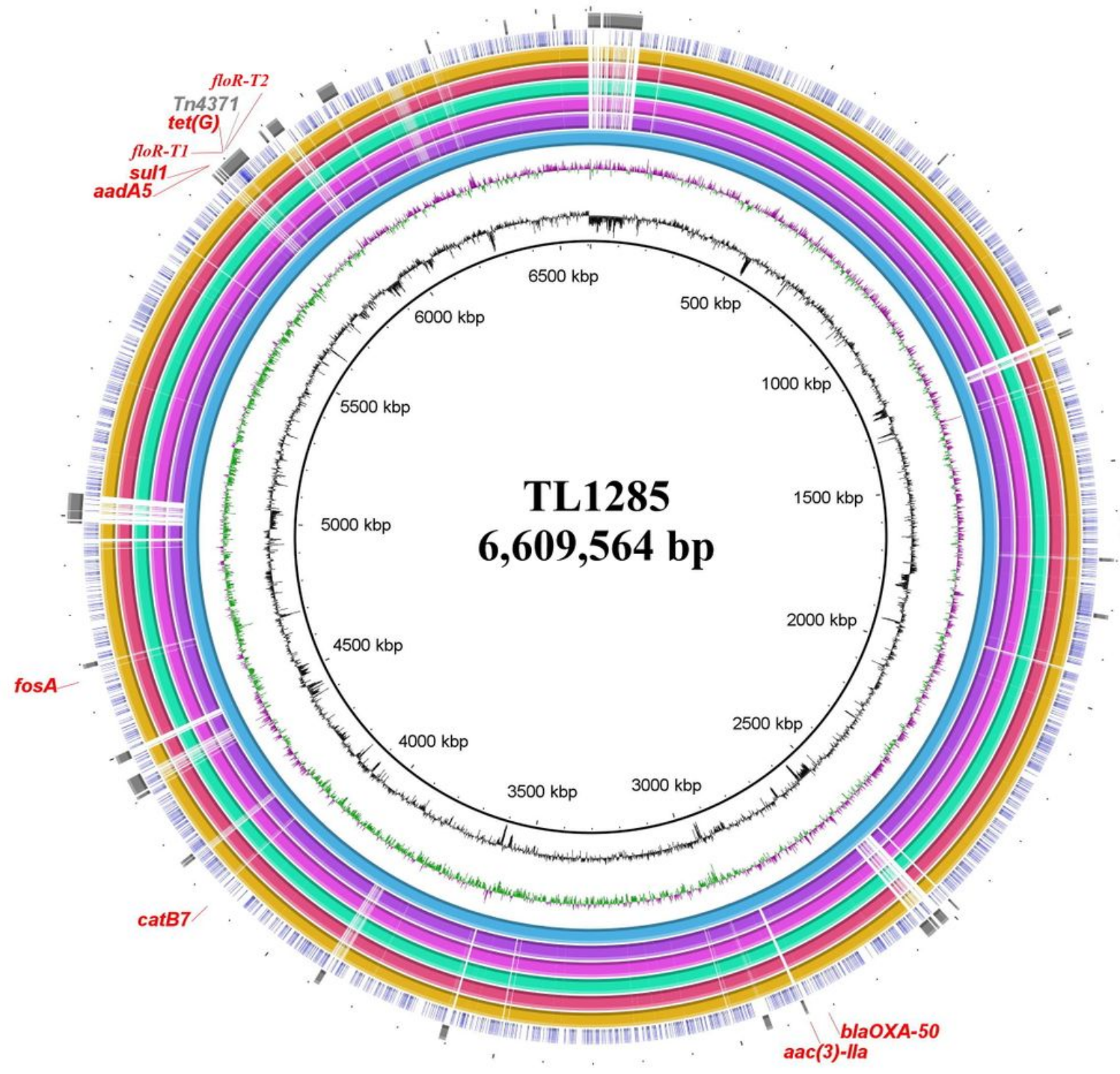

GC content

GC skew

GC skew(-)

GC skew(+)

TL1285

$100 \%$ identity

$70 \%$ identity

$50 \%$ identity

PASGNDM345

$100 \%$ identity

$70 \%$ identity

$50 \%$ identity

PASGNDM699

$100 \%$ identity

pdc-3

$70 \%$ identity

$50 \%$ identity

bcr-1 WPB099

$100 \%$ identity
$70 \%$ identity
$50 \%$ identity
WPB 100
$100 \%$ identity
$70 \%$ identity
$50 \%$ identity
WPB101
$100 \%$ identity
$70 \%$ identity
$50 \%$ identity
AR_038
$100 \%$ identity
$70 \%$ identity
$50 \%$ identity
Genomic islands

Figure 3

Sequence conservation among P. aeruginosa TL1285 and 6 other genomes carrying floR-T2. From the innermost to outermost: Circle 1 shows the scale in kb; Circles 2 and 3 represent the GC content and GC skew maps of TL1285, respectively; Circle 4 represents the genome of TL1285; Circle 5-10 represent the homologous regions of PASGNDM345, PASGNDM699, WPB099, WPB100, WPB101 and AR_038 compared to those of TL1285, while the regions without similar hits between them were left blank; Circle 11 displays the genomic islands in TL1285; Circle 12 displays the antibiotic resistance genes of TL1285. 


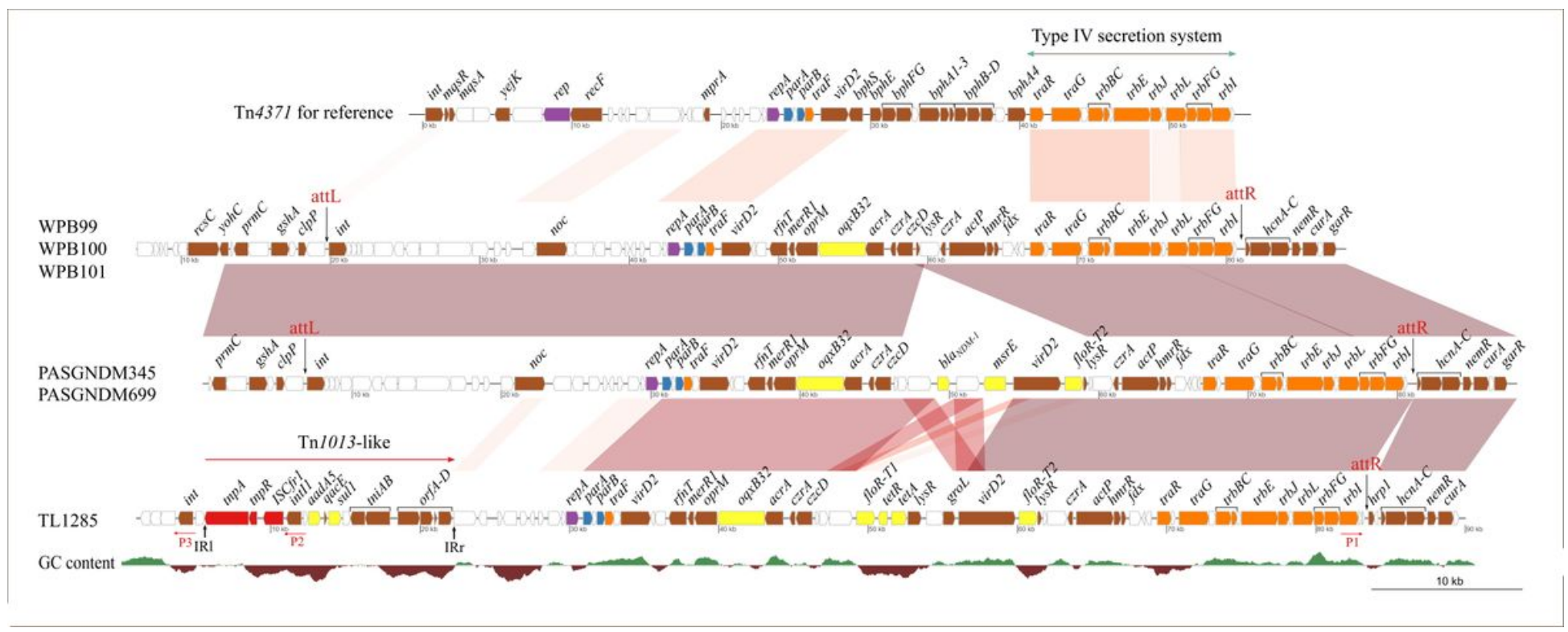

\section{Figure 4}

Comparative genomic analysis of ICE region of TL1285 and 5 other floR-T2-carrying P. aeruginosa genomes. Genes with different functions are shown in different colors: red: transposable elements; yellow: drug resistance; orange: conjugational transfer; blue: plasmid maintenance; purple: replication; brown: genes with other functions; white: hypothesis proteins.

\section{Supplementary Files}

This is a list of supplementary files associated with this preprint. Click to download.

- Figures2.jpg

- FigureS1.pdf

- TableS1.docx 